\title{
Elevated circulating stem cells level is observed one month after implantation of Carmat bioprosthetic total artificial heart
}

\section{Léa Guyonnet}

CLCC Institut Curie: Institut Curie

grégoire Detriché

APHP: Assistance Publique - Hopitaux de Paris

Nicolas Gendron

APHP: Assistance Publique - Hopitaux de Paris

Aurélien Philippe

Université de Paris Faculté de Santé: Universite de Paris Faculte de Sante

Christian Latremouille

Carmat SAS: CARMAT

\section{Lou Soret}

APHP: Assistance Publique - Hopitaux de Paris

\section{Antoine Capel}

Carmat SAS: CARMAT

\section{Christophe Peronino}

Carmat SAS: CARMAT

Piet Jansen

Carmat SAS: CARMAT

\section{Peter Ivak}

Institut klinicke a experimentalni mediciny

\section{Alain Carpentier}

APHP: Assistance Publique - Hopitaux de Paris

\section{Tristan Mirault}

APHP: Assistance Publique - Hopitaux de Paris

\section{Ivan Netuka}

Institut klinicke a experimentalni mediciny

\section{Coralie L Guerin}

Institut Curie

\section{David Smadja ( $\square$ david.smadja@me.com )}

Universite Paris Descartes https://orcid.org/0000-0001-7731-9202 


\section{Short Report}

Keywords: stem cells, endothelial progenitors, Aeson, total artificial heart, mobilization, transplantation

Posted Date: September 17th, 2021

DOl: https://doi.org/10.21203/rs.3.rs-905334/v1

License: (c) (i) This work is licensed under a Creative Commons Attribution 4.0 International License. Read Full License

Version of Record: A version of this preprint was published at Stem Cell Reviews and Reports on October 7th, 2021. See the published version at https://doi.org/10.1007/s12015-021-10270-3. 


\section{Abstract}

The Aeson ${ }^{\circledR}$ total artificial heart (A-TAH) has been developed as a total heart replacement for patients at risk of death from biventricular failure. We previously described endothelialization of the hybrid membrane inside A-TAH probably at the origin of acquired hemocompatibility. We aimed to quantify vasculogenic stem cells in peripheral blood of patients with long-term A-TAH implantation. Four male adult patients were included in this study. Peripheral blood mononuclear cells were collected before ATAH implantation (T0) and after implantation at one month (T1), between two and five months (T2), and then between six and twelve months (T3). Supervised analysis of flow cytometry data confirmed the presence of the previously identified $\mathrm{Lin}^{-} \mathrm{CD} 133^{+} \mathrm{CD} 45^{-}$and $\mathrm{Lin}^{-} \mathrm{CD} 34^{+}$with different $\mathrm{CD} 45$ level intensities. $\mathrm{Lin}^{-} \mathrm{CD} 133^{+} \mathrm{CD} 45^{-}$, $\mathrm{Lin}^{-} \mathrm{CD} 34^{+} \mathrm{CD} 45^{-}$and $\mathrm{Lin}^{-} \mathrm{CD} 34^{+} \mathrm{CD} 45^{+}$were not modulated after A-TAH implantation. However, we demonstrated a significant mobilization of $\operatorname{Lin}^{-} \operatorname{CD} 34^{+} C D 45^{\operatorname{dim}}(p=0.01)$ one month after A-TAH implantation regardless of the expression of CD133 or c-Kit. We then visualized data for the resulting clusters on a uniform manifold approximation and projection (UMAP) plot showing all single cells of the live $\mathrm{Lin}^{-}$and $\mathrm{CD} 34^{+}$events selected from down sampled files concatenated at $\mathrm{T} 0$ and $\mathrm{T} 1$. The three clusters upregulated in $\mathrm{T} 1$ are $\mathrm{CD} 45^{\mathrm{dim}}$ clusters, confirming our results. In conclusion, using a flow cytometry approach, we demonstrated in A-TAH-transplanted patients a significant mobilization of $\mathrm{Lin}^{-} \mathrm{CD} 34^{+} \mathrm{CD} 45^{\mathrm{dim}}$ in peripheral blood one month after A-TAH implantation.

\section{Introduction}

The Aeson ${ }^{\circledR}$ total artificial heart (A-TAH, Carmat, Velisy Villacoublay, France) has been developed as a total heart replacement for patients at risk of death from biventricular failure (1). The A-TAH is a biventricular, autoregulated, pulsatile, electro-hydraulically actuated heart replacement device with all components embodied in a single device, implanted in the pericardial sac (2). The surfaces in contact with the patient's blood are formed from expanded polytetrafluorethylene and bovine pericardial tissue membrane processed in glutaraldehyde (3). These types of material have demonstrated a high level of biocompatibility in various applications such as bioprosthetic cardiac valve replacement (4). A-TAH efficacy has been proven in a feasibility study and then in a pivotal study, mainly in the indication of bridge-to-transplantation, leading to recent CE market approval $(5,6)$.

We previously described, in the first three implanted patients, the histological characteristics of explanted devices (7). In electron microscopy, we found a homogeneous adherent fibrin cellular network with endothelial cell deposition on this fibrin cap (7). The endothelial phenotype was confirmed with vascular endothelial (VE)-cadherin expression and the presence of tight junctional structures observed by electron microscopy (7). The origin of these endothelial cells remains unknown. Indeed, these cells shall originate from the circulating blood, since there is no physical connection between the endothelialized hybrid membrane and the patient's blood vessels. This could be linked to circulating endothelial progenitor cells (EPCs) or very small embryonic-like stem cells (VSELs) (1). EPCs and, in particular, endothelial colonyforming cells (ECFCs) obtained in culture are vasculogenic cells in adult humans $(8,9)$. However, the 
phenotype of circulating EPCs is classically described as a subpopulation of circulating $\mathrm{CD}_{3} 4^{+}$and/or $\mathrm{CD}_{133^{+}}$stem cells expressing mainly VEGFR-2 receptor (KDR in human)(10). A multidimensional proteomic approach of circulating cells allowed us to fully characterize stem and progenitor cells in peripheral blood that could be at the origin of the endothelial cells. This extensive phenotyping enabled us to demonstrate that non-KDR cells with immaturity markers can be mobilized and confirmed the absence of KDR on circulating stem/progenitor cells (11). These mobilized progenitor cells could come from VSELs. These latter's are defined in human as lineage-negative ( $\left.\operatorname{Lin}^{-}\right), \mathrm{CD}_{133^{+}}$and/or $\mathrm{CD} 34^{+}$and CD45 ${ }^{-}$cells of small size (12-14). VSELs are able to give rise to endothelial cells and promote postischemic revascularization $(15,16)$.

The aim of the present study was to identify the distribution and phenotype of potential circulating vasculogenic stem cells in four patients after long-term A-TAH implantation.

\section{Materials And Methods}

Aeson ${ }^{\circledR}$ bioprosthetic total artificial heart (A-TAH) study design and population

The four patients presented in this study were derived from an ongoing single-arm prospective nonblinded and non-randomized study (NCT02962973) (6). The first patient was a 66-year-old who had the device implanted for 599 days and died. The second one was a 57-year-old who had the device implanted for 304 days before receiving a heart transplant. The third patient was 70 years old and had the device implanted for 271 days and was heart transplanted. The last patient was a 43-year-old who had the device implanted for 308 days before heart transplant. All patients were classified in Interagency Registry for Mechanically Assisted Circulatory Support (INTERMACS) 3 or 4 and clinical characteristics are presented in Table 1.

\section{Sampling}

Peripheral blood samples were collected on EDTA respectively before (T0), after one month (T1), between two and five months (T2), and between six and twelve months after A-TAH implantation (T3). Ficollisolated peripheral blood mononuclear cells (PB-MNCs) were isolated, aliquoted, frozen, and stored to assess the distribution of stem cells by spectral flow cytometry.

\section{Flow cytometry}

PB-MNCs samples from patients were slowly thawed and resuspended in PBS-SVF-EDTA buffer for counting in first step and resuspended at a concentration of 10 millions of cells per $\mathrm{mL}$. Cells were incubated with an anti-human antibodies cocktail of lineage (a-CD3, a-CD14, a-CD16, a-CD11b, a-CD11c, a-CD19, a-CD56), CD45-BV650 (BioLegend), CD34-PeCy7 (BioLegend), c-Kit (CD117)-PeDazzle594 (BioLegend), CD133-VioBright667 (Miltenyi Biotec) and Zombie NIR (Biolegend) for viability during 30 minutes in the dark. Cells were washed by adding PBS-SVF-EDTA buffer and centrifuged two times. 
Before acquisition, cells were fixed during 45 minutes in the dark at $4^{\circ} \mathrm{C}$. Acquisitions were performed with a three lasers Aurora spectral flow cytometer (Cytek). Unmixing was calculated and applied to samples based on reference single stained PB-MNCs controls. Data were first analyzed in a supervised way with FlowJo (FlowJo, LLC) to identify immature cells. Down sampling of $\mathrm{CD} 34^{+}$population was done for every sample. Featured on CD45, CD34, CD133 and c-Kit expressions, consecutive dimension reduction was performed by Uniform Manifold Approximation and Projection (UMAP) algorithm (17) and metaclustering was assessed by FlowSOM enabled identification of 7 clusters among the $\mathrm{CD}_{3} 4^{+}$cells using the cloud-based platform OMIQ (https://www.omiq.ai/).

\section{Statistical analysis}

Continuous data were expressed as mean of cells \pm standard error of the mean. In the univariate analysis, we determined the differences within repeated measures by a non-parametric test. A p-value of 0.05 was considered statistically significant. Statistical analysis was performed using with GraphPad Prism 9 software (GraphPad Software Inc., San Diego, USA).

\section{Results And Discussion}

PB-MNCs from four male patients were collected before A-TAH implantation (T0) and after implantation at one month (T1), between two and five months (T2), and then between six and twelve months (T3). As the A-TAH hybrid membrane has been described as being endothelialized after several months of implantation (7), we decided to explore the phenotype of circulating stem cells after implantation in order to hypothesize the cell origin of these newly formed endothelial tissues. Supervised analysis of flow cytometry data confirmed the presence of the previously identified $\operatorname{Lin}^{-} \mathrm{CD} 133^{+} \mathrm{CD} 45^{-}$and $\operatorname{Lin}^{-} \mathrm{CD} 34^{+}$with different CD45 level intensities. Lin ${ }^{-C D} 133^{+} \mathrm{CD} 45^{-}$and Lin ${ }^{-} \mathrm{CD} 34^{+} \mathrm{CD} 45^{-}$were assumed to contain VSELs and were not modulated during the period studied here. The statistical analysis showed that, among the three populations of $\mathrm{CD} 34^{+}$only the $\mathrm{Lin}^{-} \mathrm{CD} 34^{+} \mathrm{CD} 45^{\mathrm{dim}}$ was significantly increased one month after ATAH implantation in contrast to pre-implantation level $(p=0.01)$, regardless of the expression of CD133 or c-Kit. Indeed, Lin ${ }^{-C D} 34^{+} \mathrm{CD} 45^{\mathrm{dim}}$ population could be sub-divided into four categories according to the positivity of CD133 and c-Kit. When CD133 or c-Kit were positive, we always observed a significant increase in stem cells after implantation, whereas there was no significant difference after A-TAH

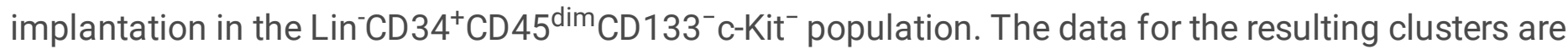
visualized on a UMAP plot in Figure 1 showing all single cells of the live Lin ${ }^{-}$and $\mathrm{CD} 34^{+}$events selected from down sampled files concatenated at T0 and T1. The algorithm proposed seven clusters. As demonstrated in Figure 1A, three of the seven clusters evidenced are upregulated in T1, in contrast to T0: clusters 7, 4, and 2. In Figure 1B, analysis demonstrated that the increase concerned the three CD 45 dim clusters, confirming our results presented in Table 2. Thus, using a flow cytometry approach, we showed a significant mobilization of $\mathrm{Lin}^{-} \mathrm{CD} 34^{+} \mathrm{CD} 45^{\mathrm{dim}}$ in peripheral blood one month after A-TAH implantation. We recently described a progressive endothelialization of the bioprosthetic hybrid membrane of the ATAH that could be at the origin of its acquired hemocompatibility (7). As there is no physical connection 
between the internal membrane of the device and the patient's blood vessels, the source of these neoendocardial cells in the A-TAH shall come from the circulating blood. Thus, the aim of this study was to identify by conventional flow cytometry approaches stem cells in blood that could be mobilized and could give rise to newly formed endothelial cells on the hybrid membrane of the A-TAH.

In the past years, $\mathrm{CD} 34^{+}$cells emerged as the most convincing cell type among those that have been evaluated for their use in cell-therapy trials and as biomarker of cardiac disease (10). CD34 ${ }^{+}$ hematopoietic stem cells with the CD45 $\mathrm{dim}$ phenotype have been proposed as a source of extra hematopoietic cells like cardiomyocytes for example (18), although this has been controversial (19). However, in human adults, we don't know with certitude the stem cell at the origin of ECFCs. Indeed, it is now admitted that ECFCs are the main human post-natal vasculogenic cells (8). ECFCs have been described to grow from circulating $\mathrm{CD} 34^{+}$cells present in adult peripheral blood, but during in vitro expansion part of the cells lose CD34. CD34 ${ }^{+}$and CD34 ${ }^{-}$ECFCs have different angiogenic properties and CD34 expression in ECFCs could be related to a specific state of endothelial phenotype (20). Their origin has been proposed in CD45 negative cells (21) but subtype of CD34 involved in ECFC differentiation is unclear (22). CD34 ${ }^{+}$cell sub-populations may be derived from VSELs. VSELs were first identified as CD 45 negative cells and characterized by their very small size (3-5 $\mu \mathrm{m}$ in diameter) in murine and human bone marrow (5-6 $\mu \mathrm{m}$ in diameter) (14). VSELs are mobilized into peripheral blood in response to injury following acute myocardial infarction (23) or critical leg ischemia (15) and we previously demonstrated that these cells trigger post-ischemic revascularization (15). Others and we have also shown VSELs ability to differentiate into endothelial cells $(15,24-26)$. Human VSELs have been described expressing CD133, but some description of human CD34+-VSELs have been done and their vascular differentiation ability confirmed (27). CD34+-VSELs can regenerate damaged organs and may solve the problems inherent in the use of controversial embryonic stem cells or induced pluripotent stem cells indeed. In our study, we did not include any size beads. However, when back gating our populations, we can assume that Lin ${ }^{-} \mathrm{CD} 133^{+} \mathrm{CD} 45^{-}$cells are only small sized cells compatible with VSELs phenotype. In contrast, Lin CD $34^{+}$CD $45^{-}$and Lin ${ }^{-C D} 34^{+}$CD $45^{\text {dim }}$ are a mix of small and large sized CD $34^{+}$cells. Lin ${ }^{-C D} 34^{+}$CD $45^{\text {dim }}$ of small size has never been specifically studied in terms of multipotent differentiation ability. Thus, we observed the mobilization of a CD $34^{+}$population with $C D 45^{\text {dim }}$ expression while the $C D 45^{\text {neg }}$ population was not mobilized. This CD $45^{\mathrm{dim}}$ population contained various-sized cells. Further study needs to evaluate the ability of $C D 45^{\mathrm{dim}} \mathrm{CD} 34^{+}$cells of small and "normal size" to give rise to endothelial cells in vitro and in vivo and validate the origin of newly formed endothelial cells on top of A-TAH hybrid membrane.

All in all, bioprosthetic A-TAH implantation allowed us to evidence the mobilization in peripheral blood of Lin ${ }^{-C D} 34^{+} C D 45^{\text {dim }}$ stem cells that could be at the origin of the endothelial recovery. In order to organize new cell-therapy trials or determine the cells at the origin of endothelial lineage in vivo further studies need to appreciate the size of stem cells that are mobilized and able to build vessels. This topic of adult stem cells at the top of the hierarchy of endothelial lineage requires research on stem cells in peripheral 
blood in other cardiovascular mobilization situations, especially organ replacement requiring cell recolonization. New multidimensional proteomic approach by flow, imaging, or mass cytometry associated with bioinformatic analysis may help to ameliorate the screening of stem cells involved in the vasculogenic process.

\section{Declarations}

\section{Acknowledgments:}

We would like to acknowledge all nurses, technicians and physicians involved in the cardiac Surgery department involved in A-TAH implantation. The central illustration figure was created with BioRender.com

\section{Ethical Approval}

Samples from the four patients in the C-TAH CE Mark clinical trial (Identifier: NCT02962973)

\section{Consent to Participate:}

All patients signed informed consent to participate to research and authorized their data publication. Consent to Publish:

All co-authors agree to publish these data.

\section{Authors Contributions:}

LG, CG and GD analyzed the data and wrote the paper. NG, AP, LS, CP, TM performed and/or analyzed the data. CL, PI, ACarpentier and IN included patients and reviewed the paper. ACapel and PJ organized clinical trials. A Carpentier is inventor of C-TAH and reviewed the paper. DMS supervised the work, analyzed the data and wrote the paper.

\section{Fundings:}

This work was supported by grants of CARMAT and PROMEX STIFTUNG FUR DIE FORSCHUNG foundation.

\section{Competing Interests:}

A Carpentier is cofounder and shareholder of CARMAT SA. DM Smadja received consulting fees from CARMAT. C Latremouille, A Capel and P. Jansen are employed by CARMAT-SAS.

\section{Availability of data and materials:}

All data are available upon request.

\section{References}


1. Han, J. J. (2021). Aeson-The Carmat total artificial heart is approved for enrollment in the United States. Artif Organs, 45(5), 445-446

2. Carpentier, A., Latremouille, C., Cholley, B., Smadja, D. M., Roussel, J. C., Boissier, E., et al. (2015). First clinical use of a bioprosthetic total artificial heart: report of two cases. Lancet, 386(10003), 15561563

3. Richez, U., De Castilla, H., Guerin, C. L., Gendron, N., Luraghi, G., Grimme, M., et al. (2019). Hemocompatibility and safety of the Carmat Total Artifical Heart hybrid membrane. Heliyon, 5(12), e02914

4. Carpentier, A. (2007). Lasker Clinical Research Award. The surprising rise of nonthrombogenic valvular surgery. Nat Med, 13(10), 1165-1168

5. Latremouille, C., Carpentier, A., Leprince, P., Roussel, J. C., Cholley, B., Boissier, E., et al. (2018). A bioprosthetic total artificial heart for end-stage heart failure: Results from a pilot study. J Heart Lung Transplant, 37(1), 33-37

6. Netuka, I., Pya, Y., Bekbossynova, M., Ivak, P., Konarik, M., Gustafsson, F., et al. (2020). Initial bridge to transplant experience with a bioprosthetic autoregulated artificial heart.J Heart Lung Transplant.

7. Smadja, D. M., Saubamea, B., Susen, S., Kindo, M., Bruneval, P., Van Belle, E., et al. (2017).

Bioprosthetic Total Artificial Heart Induces a Profile of Acquired Hemocompatibility With Membranes Recellularization. J Am Coll Cardiol, 70(3), 404-406

8. Smadja, D. M., Melero-Martin, J. M., Eikenboom, J., Bowman, M., Sabatier, F., \& Randi, A. M. (2019). Standardization of methods to quantify and culture endothelial colony-forming cells derived from peripheral blood: Position paper from the International Society on Thrombosis and Haemostasis SSC. J Thromb Haemost, 17(7), 1190-1194

9. Yoder, M. C., Mead, L. E., Prater, D., Krier, T. R., Mroueh, K. N., Li, F., et al. (2007). Redefining endothelial progenitor cells via clonal analysis and hematopoietic stem/progenitor cell principals. Blood, 109(5), 1801-1809

10. Rigato, M., Avogaro, A., \& Fadini, G. P. (2016). Levels of Circulating Progenitor Cells, Cardiovascular Outcomes and Death: A Meta-Analysis of Prospective Observational Studies. Circ Res, 118(12), 1930-1939

11. Guerin, C. L., Guyonnet, L., Goudot, G., Revets, D., Konstantinou, M., Chipont, A., et al. (2020). Multidimensional Proteomic Approach of Endothelial Progenitors Demonstrate Expression of KDR Restricted to CD19 Cells. Stem Cell Rev Rep. :In press.

12. Henon, P. (2020). Key Success Factors for Regenerative Medicine in Acquired Heart Diseases. Stem Cell Rev Rep, 16(3), 441-458

13. Lahlil, R., Scrofani, M., Barbet, R., Tancredi, C., Aries, A., \& Henon, P. (2018). VSELs Maintain their Pluripotency and Competence to Differentiate after Enhanced Ex Vivo Expansion. Stem Cell Rev Rep, 14(4), 510-524

14. Ratajczak, M. Z., Ratajczak, J., \& Kucia, M. (2019). Very Small Embryonic-Like Stem Cells (VSELs). Circ Res, 124(2), 208-210 
15. Guerin, C. L., Loyer, X., Vilar, J., Cras, A., Mirault, T., Gaussem, P., et al. (2015). Bone-marrow-derived very small embryonic-like stem cells in patients with critical leg ischaemia: evidence of vasculogenic potential. Thromb Haemost, 113(5), 1084-1094

16. Guerin, C. L., Rossi, E., Saubamea, B., Cras, A., Mignon, V., Silvestre, J. S., et al. (2017). Human very Small Embryonic-like Cells Support Vascular Maturation and Therapeutic Revascularization Induced by Endothelial Progenitor Cells. Stem Cell Rev, 13(4), 552-560

17. Becht, E., McInnes, L., Healy, J., Dutertre, C. A., Kwok, I. W. H., Ng, L. G., et al. (2019). Dimensionality reduction for visualizing single-cell data using UMAP.Nat Biotechnol.

18. Orlic, D., Kajstura, J., Chimenti, S., Jakoniuk, I., Anderson, S. M., Li, B., et al. (2001). Bone marrow cells regenerate infarcted myocardium. Nature, 410(6829), 701-705

19. Murry, C. E., Soonpaa, M. H., Reinecke, H., Nakajima, H., Nakajima, H. O., Rubart, M., et al. (2004). Haematopoietic stem cells do not transdifferentiate into cardiac myocytes in myocardial infarcts. Nature, 428(6983), 664-668

20. Tasev, D., Konijnenberg, L. S., Amado-Azevedo, J., van Wijhe, M. H., Koolwijk, P., \& van Hinsbergh, V. W. (2016). CD34 expression modulates tube-forming capacity and barrier properties of peripheral bloodderived endothelial colony-forming cells (ECFCs). Angiogenesis, 19(3), 325-338

21. Timmermans, F., Van Hauwermeiren, F., De Smedt, M., Raedt, R., Plasschaert, F., De Buyzere, M. L., et al. (2007). Endothelial outgrowth cells are not derived from CD133 + cells or CD45 + hematopoietic precursors. Arterioscler Thromb Vasc Biol, 27(7), 1572-1579

22. Case, J., Mead, L. E., Bessler, W. K., Prater, D., White, H. A., Saadatzadeh, M. R., et al. (2007). Human CD34 + AC133 + VEGFR-2 + cells are not endothelial progenitor cells but distinct, primitive hematopoietic progenitors. Exp Hematol, 35(7), 1109-1118

23. Abdel-Latif, A., Zuba-Surma, E. K., Ziada, K. M., Kucia, M., Cohen, D. A., Kaplan, A. M., et al. (2010). Evidence of mobilization of pluripotent stem cells into peripheral blood of patients with myocardial ischemia. Exp Hematol, 38(12), 1131-1421

24. Havens, A. M., Sun, H., Shiozawa, Y., Jung, Y., Wang, J., Mishra, A., et al. (2014). Human and murine very small embryonic-like cells represent multipotent tissue progenitors, in vitro and in vivo. Stem Cells Dev, 23(7), 689-701

25. Wu, J. H., Wang, H. J., Tan, Y. Z., \& Li, Z. H. (2012). Characterization of rat very small embryonic-like stem cells and cardiac repair after cell transplantation for myocardial infarction. Stem Cells Dev, 21(8), 1367-1379

26. Zhang, S., Zhao, L., Wang, J., Chen, N., Yan, J., \& Pan, X. (2017). HIF-2alpha and Oct4 have synergistic effects on survival and myocardial repair of very small embryonic-like mesenchymal stem cells in infarcted hearts. Cell Death Dis, 8(1), e2548

27. Lahlil, R., Scrofani, M., Barbet, R., Tancredi, C., Aries, A., \& Henon, P. (2018). VSELs Maintain their Pluripotency and Competence to Differentiate after Enhanced Ex Vivo Expansion. Stem Cell Rev, $14(4), 510-524$ 


\section{Tables}

Table 1: Pre-Implantation characteristics of patients implanted with A-TAH

\begin{tabular}{lllll} 
Patients & $\mathbf{1}$ & $\mathbf{2}$ & $\mathbf{3}$ & $\mathbf{4}$ \\
\hline Age (years) & 66 & 57 & 70 & 43 \\
\hline Gender & Male & Male & Male & Male \\
\hline BSA $\left(\mathbf{m}^{2}\right)$ & 2.13 & 1.89 & 2.06 & 2.36 \\
\hline Cardiac index $\left(\mathrm{L} / \mathrm{min} / \mathrm{m}^{2}\right)$ & 1.24 & 1.66 & 1.60 & 1.65 \\
\hline INTERMACS class & 4 & 3 & 3 & 3 \\
\hline Indication & DT & BTT & DT & BTT \\
\hline Support duration (days) & 599 & 304 & 271 & 308
\end{tabular}

BSA: body surface area; INTERMACS: Interagency registry for mechanically assisted circulatory support DT: Destination therapy, BTT: bridge-to-transplantation.

Due to technical limitations, Table 2 is only available as a download in the Supplemental Files section.

\section{Figures}


T0

Figure $1 \mathrm{~A}$

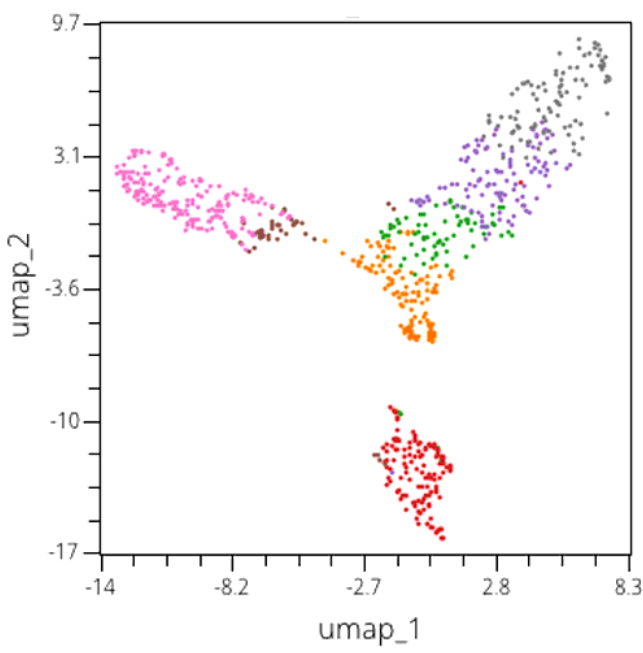

$\mathrm{T} 1$

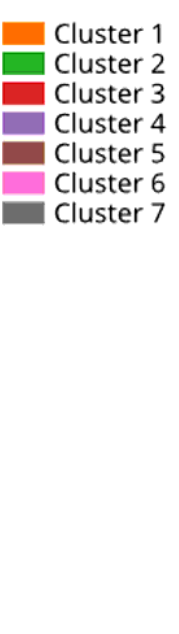

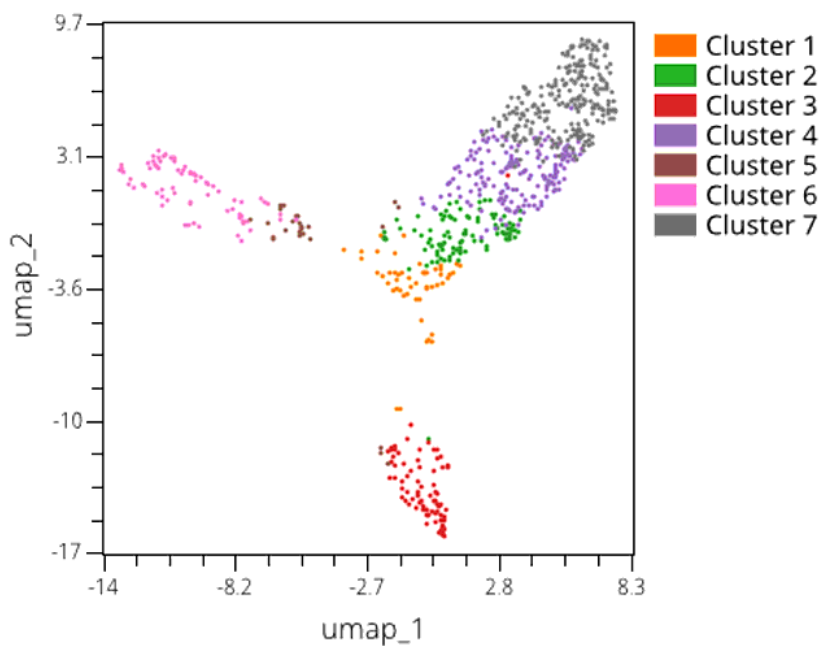

Figure 1B

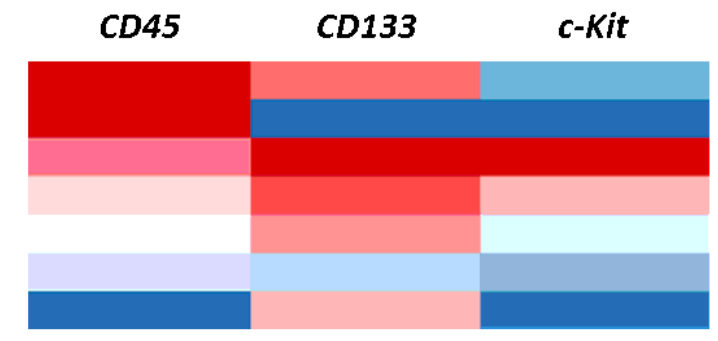

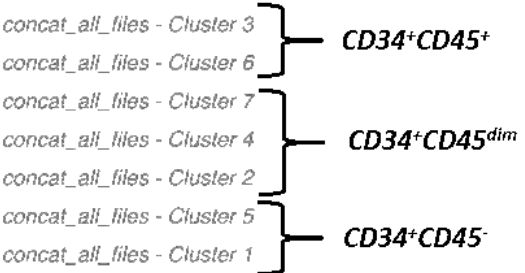

Figure 1

A- Patients concatenated CD34+ cells meta-clustering with UMAP visualization per condition, i.e .T0 before A-TAH implantation and T1 one month after implantation. B- Heatmap of CD45, CD133 and CD117 relative expression levels per cluster of $C D 34+$ patients and time points concatenated. Central illustration figure: Using a flow cytometry approach, we demonstrated in A-TAH transplanted patients a significant mobilization of Lin-CD34+CD45dim in peripheral blood one month after A-TAH implantation. This cell population could be at the origin of newly formed endothelial cells on top of hybrid membrane in Carmat bioprosthetic total artificial heart.

\section{Supplementary Files}

This is a list of supplementary files associated with this preprint. Click to download.

- Table2.docx 